\title{
Accordo di linee guida per l'utilizzazione dei filler nell'aumento del tessuto cutaneo e sottocutaneo
}

\author{
Pierfrancesco Cirillo ${ }^{1}$, Maurizio Benci ${ }^{2}$, Emanuele Bartoletti ${ }^{1}$, Carlo Bertana ${ }^{3}$ \\ Chirurgo plastico, Roma \\ Dermatologo, Firenze \\ 3 Dermatologo, Roma
}

\begin{abstract}
The presence of a lot of dermal fillers for aesthetical use and many adverse events has made necessary an accord of guidelines, among the experiences of the experts about use of this devices, that concerned the ideal characteristics of a filler, the criteria of choice (anatomical area, type of aesthetical defect ), the consolidate procedural habits, the legal aspects (clinical chart and informative consent), the behaviours in the different anatomical zones.
\end{abstract}

Keywords: filler, guidelines, consensus conference

Guidelines for the use of dermal fillers

Pratica Medica \& Aspetti Legali 2008; 2(2): 73-81

\section{INTRODUZIONE}

Molti inestetismi del viso possono essere corretti impiantando nel derma o nel sottocutaneo prodotti di origine biologica o sintetica denominati "filler". Queste sostanze hanno simili finalità terapeutiche, ma differiscono molto tra loro per caratteristiche biologiche e chimico-fisiche. L'American Society of Aesthetic Plastic Surgery (ASAPS) ha stimato nel 2004 circa 12 milioni di interventi estetici (2,1 milioni di tipo chirurgico e 9,7 milioni di tipo non chirurgico), dei quali quasi 900.000 riguardavano l'uso di sostanze per aumentare il tessuto sottocutaneo [1-4].

Lesigenza del mercato di offrire dei filler per impianti sempre più duraturi ha provocato l'immissione sul mercato europeo di numerosi prodotti, tutti regolarmente registrati con marchio $\mathrm{CE}$, composti da sostanze pressoché non metabolizzabili dall'organismo ospite. Tali filler avrebbero dovuto rispettare le norme generali di assoluta sicurezza per i pazienti, ma hanno dato numerosi effetti collaterali e complicanze più o meno importanti, legate alle caratteristiche chimico-fisiche e immu-

nologiche della sostanza iniettata, alle modalità di iniezione e alla risposta dell'organismo ricevente. Da qui è nata l'esigenza di avere delle linee guida condivisibili dalla maggioranza degli operatori del settore che avessero come scopo principale quello di fornire un ausilio professionale, elevando il livello di qualità della prestazione resa [1,5-9].

\begin{abstract}
La stesura di linee guida per I'uso dei filler mira a fornire un ausilio professionale, elevando il livello di qualità della prestazione resa, ma nel contempo si prefigge di rendere compartecipi delle finalità e della complessità della procedura i pazienti e i non addetti ai lavori
\end{abstract}

La redazione delle linee guida ha impegnato un Comitato composto da: Nicolò Scuderi, Carlo Alberto Bartoletti, Giuseppe Micali, Paolo Silvestris, Pierfrancesco Cirillo, Emanuele Bartoletti, Maurizio Benci, Carlo Bertana, che hanno coordinato la Consensus Conference Italiana sui Dermal Filler, 
proponendo a un board di esperti composto da 54 professionisti distribuiti su tutto il territorio italiano (vedi box in calce all'articolo, pag. 81), con rappresentatività bilanciata tra chirurghi plastici, dermatologi e medici estetici, e ugualmente tra universitari, ospedalieri e liberi professionisti, un questionario di 139 domande riguardanti:

- le caratteristiche ideali di un filler;

- i criteri di scelta (area anatomica, tipo di inestetismo);

- le abitudini procedurali consolidate;

- gli aspetti medico-legali (cartella clinica e consenso informato);

- i comportamenti nelle differenti zone anatomiche: pieghe nasolabiali, labbra, commissura orale, piega labiomentoniera, linea della mandibola, glabella, rughe orizzontali della fronte, sopracciglio, rughe perioculari, occhiaie, solco naso genieno, lipoatrofia del volto AIDS e non AIDS-correlata, piramide nasale, cicatrici depresse, altre sedi anatomiche.

È stato formalmente richiesto ad alcune Società Scientifiche il patrocinio ufficiale al progetto, che è stato concesso (vedi box in calce all'articolo, pag. 81). A tutti è stata inviata una richiesta di adesione al progetto, la cui risposta doveva pervenire entro e non oltre il 1 marzo 2007. Solo in seguito ad accettazione formale, è stato inviato loro un questionario da compilare e far pervenire al Comitato Organizzatore entro il 30 marzo 2007. Delle 60 richieste inviate, 54 sono state accettate.

\section{DEFINIZIONE}

Tra le varie definizioni prospettate, sembra calzante quella proposta dalla Commissione linee guida FISM ("Raccomandazioni per la partecipazione delle Società medico-scientifiche alla produzione, destinazione e valutazione di linee guida di comportamento pratico". QA 1996; 7: 77), secondo la quale le linee guida vanno intese come «raccomandazioni di comportamento clinico, prodotte attraverso un processo sistematico, allo scopo di assistere medici e pazienti nel decidere quali siano le modalità di assistenza più appropriate in specifiche circostanze cliniche» [1,5-9].

Con il termine "filler" (dall'inglese to fill = riempire) si definisce una metodica che consiste nell'iniezione nei tessuti molli di una o più sostanze, di varia natura, in grado di correggere il volume, allo scopo di ottenere miglioramenti estetici o al fine di trattare alcune affezioni cutanee e/o risolverne o migliorarne gli aspetti clinico-estetici. Il filler viene utilizzato prevalentemente in ambito clinico-estetico e trova essenzialmente indicazione nel trattamento di rughe superficiali e profonde, aumento volumetrico di distretti anatomici, correzioni di deficit cutanei congeniti o acquisiti.

\section{RAZIONALE}

La definizione di linee guida dell'utilizzo dei filler si prefigge di contribuire ad identificare e/o precisare:

- le caratteristiche e le proprietà degli agenti chimici;

- le modalità tecniche della loro applicazione;

- le misure precauzionali da attuare;

- le indicazioni cliniche e clinico-estetiche;

- i risultati raggiungibili;

- i trattamenti o le norme da rispettare nelle fasi successive al trattamento.

\section{AGENTI CHIMICI}

Lorigine del filler può essere:

- animale ( $2 \%$ di risposte del board);

- di sintesi batterica (32\%);

- di sintesi chimica (50\%).

\section{CLASSIFICAZIONE}

I filler vengono generalmente classificati in:

- permanenti;

- riassorbibili.

La definizione di semi-permanente, utilizzata spesso in passato, risulta oggi priva di significato scientifico, fuorviante e poco pratica.

\section{FASI PREPARATORIE O PRECEDENTI IL TRATTAMENTO CON I FILLER}

- Consenso informato: è consigliabile che debba essere redatto dal medico in dipendenza dal trattamento effettuato, ma la percentua- 
le di rappresentanti del board che di routine propongono un consenso informato scritto è ancora bassa $(63 \%$ del board ritiene il consenso informato di fondamentale importanza, il $25 \%$ lo ritiene importante ma non fondamentale).

- Cartella clinica: consigliabile, anche se, a tutt'oggi, per tali trattamenti, non è frequente la compilazione di una cartella.

- Fotografia: consigliabile, anche se, a tutt'oggi, per tali trattamenti, non è frequente la fotografia pre- e post-intervento (il 61\% del board non fotografa le pazienti prima del trattamento).

\section{CRITERI CLINICO-DIAGNOSTICI}

\section{CLINICI}

1. Anamnesi

generale

mirata

2. Esame clinico

ESAMI DIAGNOSTICI (se ritenuti opportuni)

\section{CRITERI CLINICI}

\section{Anamnesi}

- Generale (per valutare eventuali controindicazioni assolute).

- Mirata (per valutare eventuali controindicazioni non assolute).

\section{Esame clinico}

Occorre ovviamente prendere in considerazione:

- l'aspetto fisico generale;

- il fototipo (I-IV) secondo la classificazione di Fitzpatrick;

- il grado di fotoinvecchiamento, per scegliere la tipologia di filler;

- la densità e lo stato di attività delle ghiandole sebacee;

- il grado di lassità cutanea;

- la presenza di cicatrici ipertrofiche e/o cheloidee su tutto l'ambito cutaneo, oltre che sul viso;

- la presenza di infezioni nelle aree da sottoporre a trattamento con i filler;

- altre dermopatie;
- la corretta indicazione clinica o clinico-estetica.

Controindicazioni assolute, relative a tutti i trattamenti con filler

- Iniezione intra-vascolare.

- Pazienti con tendenza a sviluppare cicatrici cheloidi.

- Durante la gravidanza e l'allattamento, per carenza di studi approfonditi ( $95 \%$ del board).

- Pazienti con ipersensibilità specifiche accertate.

- Trattamento su cute lesa.

- Trattamento su cute con problemi infettivi e/o infiammatori.

- Terapie in atto, controindicate per supposti rallentamenti e/o ritardi della fase riparativa.

- Malattie autoimmunitarie, per rischio elevato di:

- infezioni;

- rallentamento dei processi riparativi.

\section{Controindicazioni non assolute relative} a tutti i trattamenti con filler

- Presenza di filler non noti.

- Pregressa radioterapia, limitante la riepitelizzazione a partenza annessiale.

- Diatesi fibroblastica.

La presenza di filler pregressi non noti è motivo sufficiente per evitare l'uso di altri filler $(92 \%$ del board) (Figura 1).

È sempre necessario valutare comunque che $i$ candidati al trattamento con i filler siano animati da aspettative realistiche e manifestino adeguata

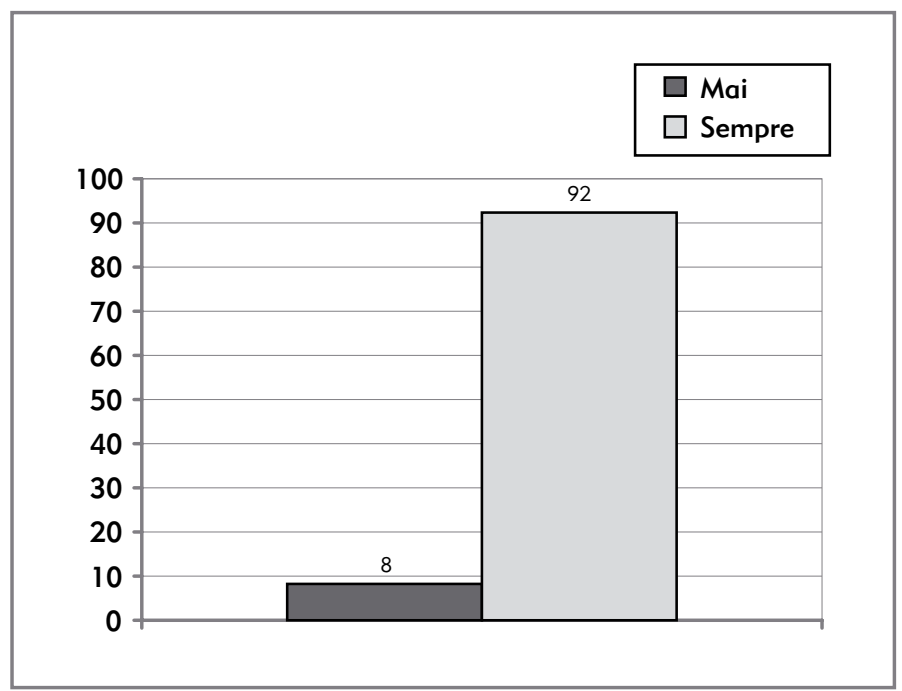

Figura 1. La presenza di filler pregressi non noti, è motivo sufficiente per evitare altri filler? (percentuali di risposta del board) 


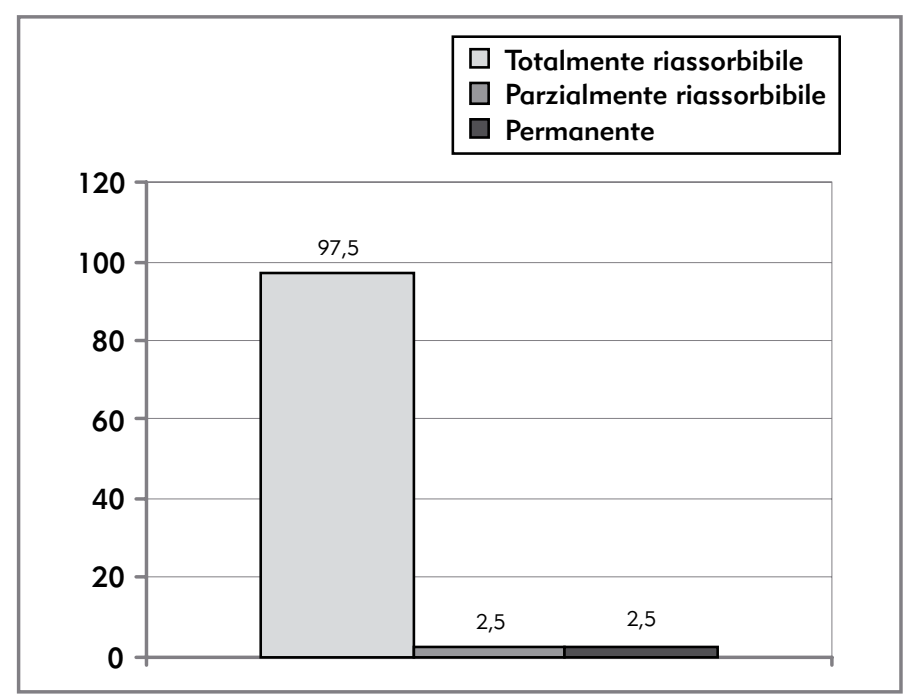

Figura 2. A prescindere dalla natura specifica del prodotto, è oramai opinione concorde che la scelta debba cadere su un filler a completa riassorbibilità (percentuali di risposta del board)

disponibilità psicofisica a tollerare il trattamento e la convalescenza.

In pazienti ansiosi, può essere prudente praticare un test anche per i filler che non contemplano tale obbligo, per valutare (ma non è un rilievo da ritenere sicuramente predittivo e probante) una eventuale ipersensibilità specifica.

È anche indispensabile verificare la disponibilità e la capacità del paziente ad effettuare eventuali medicazioni o trattamenti specifici successivamente al trattamento, come ad esempio il camoufflage, anche per tempi lunghi, in caso di ematomi persistenti, di reazioni infiammatorie (arrossamenti, edemi, eritemi).

Il paziente, per poter essere sottoposto a una terapia iniettiva con dermal filler, deve avere una età minima di 18 anni; al di sotto dei 18 anni è possibile trattare il paziente solo con il consenso dei genitori ( $92 \%$ del board).

Scelta dei materiali: a prescindere dalla natura specifica del prodotto, è oramai opinione concorde che la scelta debba cadere su un filler a completa riassorbibilità $\mathbf{( 9 7 , 5 \% ~ d e l ~ b o a r d ) ~ ( F i g u r a ~ 2 ) . ~}$

\section{TEST PRE-TRATTAMENTO}

- Obbligatori 2 test nella regione volare dell'avambraccio, per l'utilizzo di filler a base di collagene bovino; il primo, con periodo di osservazione di non meno di 30 giorni, il secondo con periodo di osservazione di non meno di 7 giorni.
- Facoltativi i test per i collageni di derivazione porcina o umana e per le altre tipologie di filler, da consigliare in pazienti con anamnesi positiva a patologie su base auto-immunitaria.

- Anche con i filler riassorbibili il paziente non ritorna mai allo stato iniziale; quindi reversibilità non significa restitutio ad integrum. Il $60 \%$ del board degli esperti ha dichiarato che il paziente non ritorna allo stato iniziale.

\section{SCELTA DI DISINFETTANTI ADEGUATI, SULLA BASE DELLA TIPOLOGIA DEL FILLER}

Esiste una incompatibilità accertata tra l'acido ialuronico e i disinfettanti a base di sali di ammonio quaternario.

\section{PRECAUZIONI PROCEDURALI}

- Porre il paziente in posizione reclinata di $30^{\circ}$.

- Fare precedere il trattamento dall'apposizione di ghiaccio o di compresse fredde sulla zona da trattare, da utilizzare anche dopo il trattamento, per diminuire il rischio di ematomi.

- Per il trattamento di zone anatomiche molto sensibili o in pazienti particolarmente sensibili agli stimoli dolorosi, è consigliabile l'uso di anestetici topici, fino all'uso di anestesie loco-regionali e/o tronculari.

- In dipendenza dal filler utilizzato, soprattutto per quelli a maggiore densità, è consigliata l'applicazione di un massaggio, nella zona trattata, avvisando il paziente che, a fronte di una maggiore distribuzione del prodotto per un risultato naturale, aumenta il rischio di formazione di ematomi.

- Se rimane nella siringa una quantità di materiale, il medico non può conservare e riutilizzare il prodotto aperto e utilizzato parzialmente, ma deve gettare il prodotto rimasto o utilizzare il prodotto rimasto per un'altra area durante lo stesso trattamento. 


\section{ASPETTI RELATIVI ALLA SICUREZZA}

\section{REQUISITI DELL'OPERATORE}

Obbligatori:

- laurea in medicina e chirurgia ed abilitazione professionale.

Auspicati:

- specializzazione in dermatologia o in chirurgia plastica;

- perfezionamento in medicina estetica tramite corsi di studio accreditati.

Il medico deve comunque avere piene conoscenze dell'anatomia, dell'istologia e della fisiopatologia della cute e del tessuto sottocutaneo e, più in specifico, dei seguenti temi:

- anatomia cutanea delle unità cosmetiche del viso;

- differenze strutturali e funzionali delle varie aree corporee;

- fisiopatologia del danno fotoindotto;

- varie fasi della riparazione tissutale:

- processi coagulativi e infiammatori;

- angiogenesi;

- formazione di tessuto di granulazione;

- riepitelizzazione;

- rimodellamento del collagene.

È fondamentale che il medico abbia esperienza clinico-pratica, maturata durante la specialità in dermatologia o in chirurgia plastica, e/o abbia frequentato corsi di perfezionamento accreditati teorico-pratici, per acquisire la conoscenza di tecniche e caratteristiche fisico-chimiche dei materiali in uso.

Secondo il board degli esperti, l'uso dei dispositivi medici denominati filler deve avvenire esclusivamente in struttura medica attrezzata e autorizzata, da parte di personale sanitario medico esperto (100\% del board).

Dopo il trattamento il paziente dovrà:

- evitare attività fisica intensa per 6 ore;

- evitare il sole e/o le attività al freddo fino a che il rossore e/o il gonfiore non scompare;

- evitare il sole finché è evidente il livido;

- evitare prodotti per la pelle che contengano per es. retinoidi o alfa-idrossiacidi per il giorno successivo;

- usare una crema antibiotica se prescritta dal medico;

- usare una crema antiedema se prescritta dal medico;

- usare una crema per ridurre i lividi (eparinoide o altro) se prescritta dal medico;

- evitare alcolici;
- evitare farmaci antinfiammatori non steroidei;

- evitare il trucco nelle 12 ore successive.

\section{RISCHI CONTROLLABILI}

- Evitare di effettuare ipercorrezioni, laddove le caratteristiche del prodotto non lo consentano.

- I pazienti in trattamento continuo con farmaci anticoagulanti devono essere allertati circa un maggior rischio di formazione di ematomi (75\% del board).

- I pazienti che fanno un uso sporadico di acido acetilsalicilico o simili devono sospendere, ove possano, tale assunzione almeno 7 giorni prima del trattamento onde evitare esclusivamente il rischio di ematomi.

- Il periodo mestruale può influenzare la comparsa di maggiori edemi o di piccoli ematomi.

Secondo il board degli esperti le aree anatomiche in cui, utilizzando un filler riassorbibile, si assiste $\mathrm{a}$ un riassorbimento più veloce sono la bocca $(46 \%)$ e il solco naso-labiale (38\%).

I soggetti fumatori potrebbero avere un riassorbimento più veloce, al pari dei pazienti che effettuano una forte, continua e non protetta fotoesposizione naturale $o$ artificiale

\section{EFFETTI SECONDARI}

- Reazioni infiammatorie (arrossamento, edema, eritema, ecc.) più o meno associate a prurito. Tali effetti possono persistere anche per 7 giorni.

- Dolore al tatto nella zona trattata.

- Ematomi.

- Ipercromia o ipocromia nella zona di iniezione.

- Formazione di modularità nella zona trattata.

- Scarso effetto riempitivo in dipendenza delle caratteristiche del tessuto trattato.

- Rari casi di necrosi, soprattutto in regione glabellare. 
- Ascessi, granulomi.

- Ipersensibilità specifiche immediate o ritardate [10-15].

\section{RIPETIBILITÀ DEL TRATTAMENTO CON I FILLER}

La ripetibilità può essere programmata a seconda del materiale utilizzato e/o delle evidenze cliniche o clinico-estetiche del paziente, ma è estremamente dipendente dalle caratteristiche fisiologiche del tessuto trattato, dalle abitudini del paziente (fumo, dimagramento, fotoesposizione, ecc.), dalle aree anatomiche trattate, e la riassorbibilità del prodotto non può essere prevista se non con dati statistici, che hanno valore puramente indicativo

\section{REQUISITI NECESSARI DEL DISPOSITIVO MEDICO FILLER}

I dermal filler sono dispositivi medici, i cui requisiti generali essenziali, rivolti alla sicurezza del prodotto, sono relativi a (Direttive di riferimento D. Lgs. 507/97):

- sicurezza e salute dei pazienti e utilizzatori;

- analisi dei rischi.

- minimizzazione dei rischi associati all'uso;

- garanzia delle prestazioni del dispositivo assegnate dal fabbricante;

- inalterabilità delle caratteristiche del dispositivo durante l'uso, il trasporto e l'immagazzinamento;

- sono classificati come dispositivi di tipo chirurgico (all.IX D. Lgs. 46/97): «Dispositivi che penetrano attraverso la superficie del corpo sia nel contesto di un intervento chirurgico (bisturi) sia al di fuori di tale contesto (ago di siringa)".

A seconda delle caratteristiche fisico-chimiche del prodotto, questi può essere classificato come:

- classe IIb: dispositivi a rischio medio/alto, quali alcuni dispositivi non attivi (specie invasivi) e i dispositivi attivi che interagiscono con il corpo in maniera pericolosa (ed è il caso dei filler permanenti);

- classe III: dispositivi ad alto rischio, quali gran parte dei dispositivi impiantabili, quelli contenenti farmaci o derivati animali e alcuni dispositivi che interagiscono sulle funzioni di organi vitali (è il caso dei filler riassorbibili) $[3,4,10-15]$.

\section{REQUISITI AUSPICABILI DEL DISPOSITIVO MEDICO FILLER}

I requisiti che la Consensus Conference istituita ha reputato importanti, per ciò che riguarda le caratteristiche auspicabili oggi e necessarie domani di un filler, sono quelli di seguito elencati.

Le caratteristiche primarie del dispositivo medico iniettabile denominato filler devono essere (Tabella I):

- non sensibilizzante né a breve, né a lungo termine;

- totalmente riassorbibile;

- non cancerogeno;

- atossico;

- non immunogenico;

- non migrante;

- di consistenza il più simile possibile al tessuto ospite;

- con un'ampia e probante documentazione scientifica.

La biocompatibilità della molecola che forma il dispositivo medico filler deve possedere come requisito precedente all'immissione in commercio uno studio multicentrico della durata di 3 anni affidato a centri accreditati pubblici e privati, con pubblicazione dei risultati su rivista scientifica accreditata, preferibilmente indexata (95\% del board).

Per essere considerato affidabile e sicuro, il filler deve possedere, come requisito precedente all'immissione in commercio, uno studio multicentrico della durata di 3 anni affidato a centri accreditati pubblici e privati, con pubblicazione dei risultati su rivista scientifica accreditata, preferibilmente indexata (65\%).

La pubblicità di un filler deve essere:

- veritiera;

- aderente alle caratteristiche del prodotto;

- esente da espressioni quali: "sicuro", "innocuo", "esente da rischi";

- esente da dichiarazioni fuorvianti in materia di efficacia;

- autorizzata dal Ministero della Salute.

Il dispositivo medico iniettabile denominato filler deve essere posto in commercio sotto forma di siringhe pre-riempite sigillate in busta chiusa (100\%).

Il dispositivo medico denominato filler deve essere monouso (98\%).

Il dispositivo medico iniettabile denominato filler deve essere munito di targhette multiple adesive con i dati riferiti al nome della sostanza, al lotto di produzione e alla data di scadenza, di foglio informativo dettagliato circa le caratteristiche, le indicazioni, i modi d'uso, le precauzioni, gli effetti 
collaterali previsti, le controindicazioni, le interazioni eventuali, i dosaggi consigliati e i modi di conservazione della sostanza (92\%).

Un filler deve essere tracciabile. Con tracciabilità si intende la costante possibilità per il fabbricante di rintracciare, per qualsiasi evenienza che lo renda necessario, i dispositivi prodotti per confrontarli ai lotti di controllo ed, eventualmente, applicare le misure correttive necessarie e informare le Autorità Competenti (Art. 10 del D. Lgs. 46/97).

I dermal filler cosiddetti "No Test" possono fregiarsi di tale qualità solo dopo aver pubblicato rigorosi studi sulla sicurezza a lungo termine (almeno 3 anni) della molecola costitutiva da parte di istituti scientifici riconosciuti (75\%).

La categoria dei dermal filler è estremamente variegata sia per caratteristiche chimiche (collagene, acido ialuronico libero e cross-linkato, agarosio, idrossiapatite, ecc.) che per quelle fisiche (densità variabili per usi variabili, filler composti, ecc.). Tali peculiarità si traducono in un differente grado di difficoltà d'uso: sarebbe utile che il fabbricante evidenziasse che, per filler particolari, è indicato un utilizzatore esperto $[3,4,10,16-18]$.

\section{AVVERTENZE}

1. La stesura delle linee guida si basa sullo stato delle attuali conoscenze e riflette dati desunti dalla letteratura scientifica di riferimento. In ogni caso i dati vanno sempre analizzati e interpretati attentamente, con adeguato senso critico

2. Studi successivi potranno indurre modifiche o cambiamenti delle conclusioni o raccomandazioni riportate in questo documento

3. II rispetto delle linee guida non assicura in ogni caso la certezza di un trattamento sicuramente soddisfacente ed esente da rischi

4. La valutazione definitiva, relativa alle caratteristiche e alle proprietà di ogni specifica procedura con i filler, compete in ogni caso al medico, sulla base della valutazione clinica e delle indicazioni relative al singolo paziente, nella definita circostanza temporale, coincidente con il momento dell'effettuazione del trattamento

5. L'utilizzo di filler impegna sul piano etico, culturale e medico-legale gli utilizzatori e i produttori

\begin{tabular}{|l|c|}
\hline \multicolumn{1}{|c}{ Caratteristiche del filler } & $(\%)$ \\
\hline Anallergico & 80 \\
\hline Non sensibilizzante né a breve, né a lungo termine & 87 \\
\hline Non cancerogeno & 92 \\
\hline Atossico & 92 \\
\hline Non immunogenico & 87 \\
\hline Di lunga durata & 40 \\
\hline Permanente & 2 \\
\hline Non migrante & 82 \\
\hline Di facile utilizzo & 50 \\
\hline Di facile produzione & 15 \\
\hline Di consistenza il più simile possibile al tessuto ospite & 57 \\
\hline Con un giusto rapporto prezzo-qualità-durata & 42 \\
\hline Con un'ampia e probante documentazione scientifica & 85 \\
\hline
\end{tabular}

Tabella I. Le caratteristiche primarie del dispositivo medico iniettabile denominato filler secondo le risposte del board (indicate in \%) 


\section{BIBLIOGRAFIA}

1. Matarasso SL, Carruthers JD, Jewell ML. Restylane Consensus Group. Consensus recommendations for soft-tissue augmentation with nonanimal stabilized hyaluronic acid (Restylane). Plast Reconstr Surg 2006; 117: 3S-34S

2. Benci M. Filler, normative comunitarie per il loro utilizzo. Kosmè 2002; 2: 49-50

3. Cirillo P, Benci M, Silvestris P, Pepe NR, Amaturo A. Atti del Congresso Nazionale SICPRE: Relazione-denuncia sulla carenza legislativa in materia di dispositivi medico chirurgici, in relazione soprattutto ai filler permanenti, e raccolta di complicanze. Pisa: 16-18 settembre 2004

4. Cirillo PF, Silverstris P, Benci M. Filler riassorbibili o non riassorbibili? Dermatologia Ambulatoriale 2005; 1-2: 14-17

5. Drake LA, Dinehart SM, Goltz RW, Graham GF, Hordinsky MK, Lewis C et al. Guidelines of care for chemical peeling. Guidelines/ Outcomes Committee: American Academy of Dermatology.J Am Acad Dermatol 1995; 33: 497-503

6. Drake LA, Ceilley RI, Cornelison RL, Dinehart SM, Dorner W, Goltz RW et al. Guidelines of care for office surgical facilities. Part I. J Am Acad Dermatol 1992; 26: 763-5

7. Drake LA, Ceilley RI, Cornelison RL, Dinehart SM, Dorner W, Goltz RW et al. Guidelines of care for office surgical facilities. Part II. Self-Assessment checklist. J Am Acad Dermatol 1995; 33: 265-70

8. Griffiths CE. The british association of dermatologists guidelines for the management of skin disease. Br J Dermatol 1999; 141: 396-7

9. Labrini G, Guerriero G, Landi FL, Teofoli P, Cirfera V. Peeling chimici. Linee guida. Dermatologia Ambulatoriale 2006; 14: 2

10. Benci M, Cirillo P, Bertana C, Fabianelli F. Complicanze da iniezione di filler riassorbibili e non riassorbibili e loro gestione. In: AA.VV. Cosmetologia Medica e Medicina degli Inestetismi Cutanei. Paris: Elsevier SAS, 2006

11. Grippando FR, Sparvieri C, Rossi A, Onesti MG, Scuderi N. Ultrasound assisted liposuction for the removal of siliconomas. Scand J Plast Reconstr Surg Hand Surg 2004; 38: 21-6

12. Onesti MG, Renzi LF, Paoletti F, Scuderi N. Use of polilactic acid in face lipodystrofy in HIV positive patients undergoing treatment with antiretroviral drugs (HAART). Acta Chir Plast 2004; 46: 12-5

13. Onesti MG, Mazzocchi M, Paoletti F, Tanzilli P. La correzione dei dimorfismi nei pazienti con infezione da HIV in trattamento con terapia antiretrovirale. Giornale Italiano di Malattie Infettive 2005; 11: 28-31

14. Onesti MG, Buccheri E, Martano A, Papasso A, Madonna Terracina FS, Mazzocchi M, Scuderi N. Silicone liquido iniettabile: revisione della letteratura e nostra esperienza. Dermatologia Clinica 2006; 4: 211-5

15. Dessy LA, Mazzocchi M, Monarca C, Onesti MG, Scuderi N. Combined transdermal scopolamine and botulin toxin A to treat a parotid fistul after face-lift in a patient with siliconomas. Int J Oral Maxillofac Surg 2007; 25

16. Rohrich RJ. Semipermanent and permanent dermal/subdermal fillers supplement. Plast Reconstr Surg 2006; 118: 1S-3S

17. Anonymous. Guidelines of care for soft tissue augmentation: gelatin matrix implant. American Academy of Dermatology. J Am Acad Dermatol 1996; 34: 695-7

18. Benci M, Lorusso B, Salti G, Cirillo P, Lotti T. About the safety an efficacy of the available fillers: four granulomatous reactions due to permanent filling substances in the perioral region.J Eur Acad Dermatol Venereol 2002; 164: 16

19. Bertana C. Acido polilattico: linee-guida. Dermatologia Ambulatoriale 2005; 13: 1-2

\section{CONSENSUS CONFERENCE ITALIANA SUI DERMAL FILLER}

Il Comitato organizzatore:

N. Scuderi, C.A. Bartoletti, G. Micali, P. Silvestris, P. Cirillo, E. Bartoletti, M. Benci, C. Bertana 


\section{CONSENSUS CONFERENCE ITALIANA SUI DERMAL FILLER}

II Comitato organizzatore

N. Scuderi, C.A. Bartoletti, G. Micali, P. Silvestris, P. Cirillo, E. Bartoletti, M. Benci, C. Bertana

Presidente SICPRE

Guido Molea

Delegati SICPRE

Elio Caccialanza, Maurizio Valeriani, Stefano Bruschi

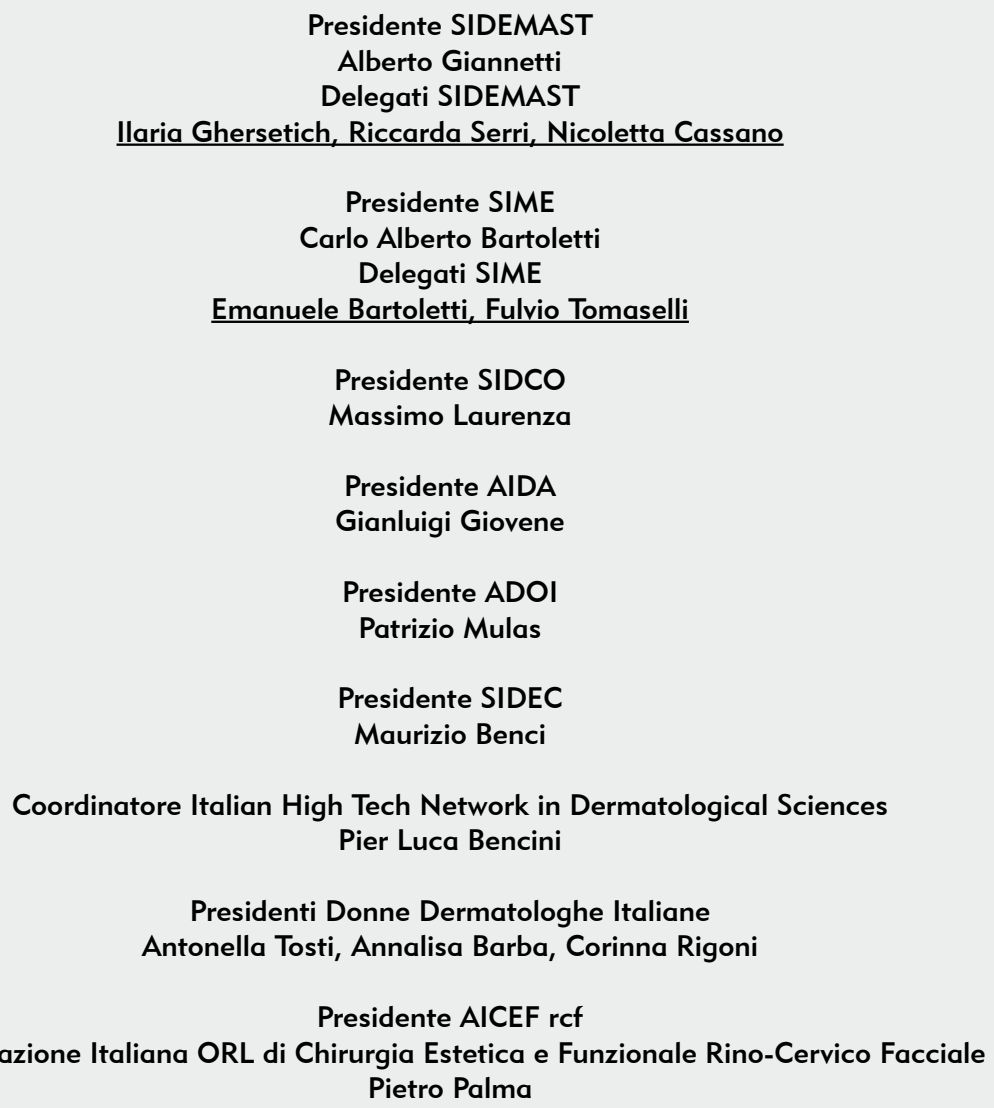

\section{Board degli esperti:}

Atzori Laura, Bartoletti Emanuele, Benci Maurizio, Bencini Pier Luca, Bertana Carlo, Botti Giovanni, Bovani Bruno, Bracaglia Roberto, Bruschi Stefano, Caccialanza Elio, Caputo Maria Grazia, Cecchini Giammatteo, Cirillo Pierfrancesco, Corazzol Annachiara, Corbo Andrea, Dal Canton Marco, De Padova Maria Pia, Dorato Dario, Fabianelli Fernanda, Faga Angela, Forte Riccardo, Gandolfi Eugenio, Gennai Alessandro, Ghersetich Ilaria, Izzo Giuseppe Maria, Klinger Marco, Lanzaro Florindo, Londei Giorgio, Maggiori Sergio, Melandri Davide, Muti Egle, Onesti M. Giuseppina, Pelle Ceravolo Mario, Petrini Nerella, Portincasa Aurelio, Rigoni Corinna, Romagnoli Marina, Rusciani Sforza Luigi, Saccomanno Flavio, Salti Giovanni, Scuderi Nicolò, Serra Visconti Anadela, Serri Riccarda, Signorini Massimo, Siliprandi Luca, Stagno D'Alcontres Francesco, Tantussi Franca, Tedeschi Aurora, Tommaselli Fulvio, Traili Fiammetta, Trainotti Claudio, Trocchi Gloria, Venturo Nicola, Vignoli Maurizio 